\title{
Fabrication and physical properties of permalloy nano-size wires
}

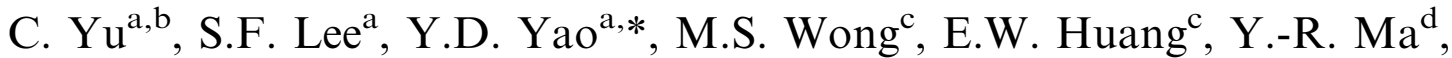 \\ J.L. Tsai ${ }^{\mathrm{a}}$, C.R. Chang ${ }^{\mathrm{b}}$ \\ ${ }^{a}$ Institute of Physics, Academia Sinica, Taipei 115, Taiwan \\ ${ }^{\mathrm{b}}$ Department of Physics, National Taiwan University, Taipei 106, Taiwan \\ ${ }^{\mathrm{c}}$ Department of Materials Science and Engineering, National Dong Hwa, University, Hualien 974, Taiwan \\ ${ }^{\mathrm{d}}$ Department of Physics, National Dong Hwa University, Hwalien 974, Taiwan
}

\begin{abstract}
Nano-size $\mathrm{NiFe}$ wires with patterned shapes in half-ring-in-series, octagon-in-series, and zigzag-in-series configurations were fabricated. Their magnetoresistance was studied below room temperature and their magnetic domain images were investigated at room temperature by a magnetic force microscope. In general, we have experimentally demonstrated that the variation of the magnetoresistance of our patterned nano-size wires can be related to different domain configurations and explained by the domain switching effect. The number of magnetic domain walls in our patterned wires can be controlled by the shape anisotropy and the size of each section of patterns that form the wires.
\end{abstract}

(C) 2002 Elsevier Science B.V. All rights reserved.

PACS: 73.61.-r; 75.60.Ch; 75.70.Ak

Keywords: Nano-size wires; Magnetoresistance; Magnetic domain

\section{Introduction}

Fabrication techniques for nano-sized dots, wires, rings, and chains have been extensively developed during recent years [1-4]. The studies of magnetic patterned nano-structures are currently an active field of research because of its possible technological applications in very high density terabit storage [5]. Their various physical proper-

\footnotetext{
*Corresponding author. Tel.: +886-2-27899617; fax: + 8862-27899636.

E-mail address: phyao@gate.sinica.edu.tw (Y.D. Yao).
}

ties, e.g. magnetic domain and transport properties, microstructure, and shape anisotropy, etc. are very sensitive to the process of fabrication. For example, the magnetic domain configurations can be most easily controlled by the shape anisotropy. These configurations are governed by the direct exchange interaction and the dipole interaction $[6,7]$ and are sensitive to shape fluctuations and edge roughness. Magnetic structures used for magnetic storage can have a complex remnant state, which influences the moment switching process. For the purpose of high-density magnetic storage media, well-defined and reproducible 
remnant states and switching processes are important. Recently, a vortex state in mesoscopic ferromagnetic rings was demonstrated to be one possible way to overcome these complications $[3,5,8,9]$. By removing the energetic vortex core in circular disks, it was found that ring shaped materials have well-defined magnetic states $[9,10]$, which were termed onion state and vortex state. The onion state has a mirror image magnetic configuration with respect to the two halves on the ring cut by a diameter, and the vortex state has a flux closure configuration. The onion state under appropriate experimental condition can be switched into the reversed onion state via a simultaneous movement of the domain walls, without any complicated intermediate states. Detailed studies on the magnetization reversal in a single ring magnet, however, are still needed.

Transport measurement is another important issue in magnetic structures, especially the domain wall magnetoresistance. Lots of efforts have been spent on how domain wall affects the magnetotransport both in thin wires $[2,10,11]$ and thin films [12]. Shape anisotropy and magnetocrystalline anisotropy are two of the controlling factors in determining the domain structures [12,13]. In this investigation, we report the fabrication and physical properties of permalloy nano-size wires with patterned shape in half-ring-in-series, octagon-in-series, and zigzag-in-series on $\mathrm{Si}\left(\begin{array}{lll}1 & 0 & 0\end{array}\right)$ wafers with a $100 \mathrm{~nm} \mathrm{SiO}_{2}$ buffer layer.

\section{Experimental}

The permalloy nano-size wires with patterned shape in half-ring-in-series, octagon-in-series, and zigzag-in-series on $\operatorname{Si}\left(\begin{array}{lll}1 & 0 & 0\end{array}\right)$ wafers with $100 \mathrm{~nm}$ $\mathrm{SiO}_{2}$ buffer layer were fabricated by means of electron-beam lithography and lift-off techniques. Electron-beam resist, polymethyl methacrylate-comethacrylic acid and copolymer were spin coated onto the substrate separately. There was a $30 \mathrm{~min}$ baking process for samples at $135^{\circ} \mathrm{C}$. After the electron-beam writing process of the octagon patterns, MIBK (Metgyl Iso Butyl Keyton) and IPA (Iso Propyl Alcohol) were used to develop the patterns. $\mathrm{Ni}_{80} \mathrm{Fe}_{20}$ films were deposited by $\mathrm{DC}$ magnetron sputtering. For the half-ring-in-series wires, the width and thickness were 200 and $25 \mathrm{~nm}$, respectively. The diameter of each half-ring was $4000 \mathrm{~nm}$ and the total length between two electrodes was roughly $24,000 \mathrm{~nm}$. For the octagon-inseries wires, the size of each octagon unit is plotted in Fig. 1(a). The thickness and length of the wires was fixed at 40 and $50,000 \mathrm{~nm}$. Zigzag wires with a line width of $500 \mathrm{~nm}$ and a pitch length of $8000 \mathrm{~nm}$ were fabricated between two gold pads separated by $50,000 \mathrm{~nm}$. The total length of the zigzag wires is roughly $73,000 \mathrm{~nm}$, and the number of corners of the zigzag wire is 12 .

Resistance measurements were made by a standard four-probe technique in a variable temperature and magnetic field platform (PPMS, Quantum Design Model 6000) in the temperature range between 10 and $300 \mathrm{~K}$. The current used for the resistance study was always small to avoid any local heating that could damage the wires. The magnetic field was applied always in the in-plane direction. A maximum magnetic field of $20 \mathrm{kOe}$ was used, and detailed investigations were performed from -2000 to +2000 Oe. The magnetic domain observations were made by a real-time magnetic force microscope (MFM) equipped with magnetic fields up to $1000 \mathrm{Oe}$.

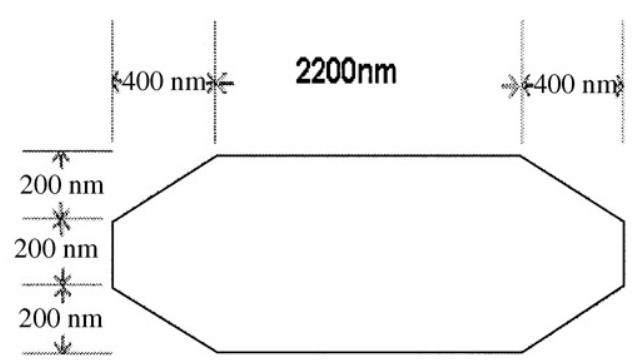

(a)

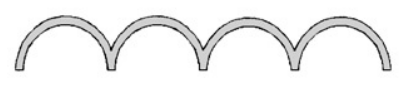

(b)

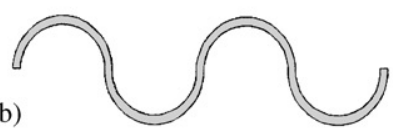

Fig. 1. Specification of (a) an octagon unit in the octagon-inseries wire, and (b) half-ring shape and S-shape in the half-ringin-series wire. 


\section{Results and discussion}

The permalloy nano-size wires with patterned shapes in the half-ring-in-series are shown in Fig. 1(b). The line width and thickness were 200 and $25 \mathrm{~nm}$, respectively. At first, we examine the angular dependence of the magnetoresistance by rotating the samples in an in-plane magnetic field of $6000 \mathrm{Oe}$ at room temperature. We know that the anisotropic magnetoresistance in a ferromagnetic material is due to spin-orbit coupling; it depends on the angle $\cup$ between the current and the local magnetization in the following manner: $\rho=\rho_{\perp}+\left(\rho_{\|}-\rho_{\perp}\right) \cos ^{2} \theta$, where $\rho_{\|}$and $\rho_{\perp}$ are the resistivities for $\theta=0^{\circ}$ and $\theta=90^{\circ}$, respectively. From symmetry considerations, for a S-shape halfring-in-series wire with infinitely thin width, the angular dependent magneto-resistance (AMR) effect should vanish at the saturated magnetization field. The AMR ratio for the S-shape wire, as shown in Fig. 2(a), is about $0.02 \%$, quite small. However, for the half-shape wire, as shown in
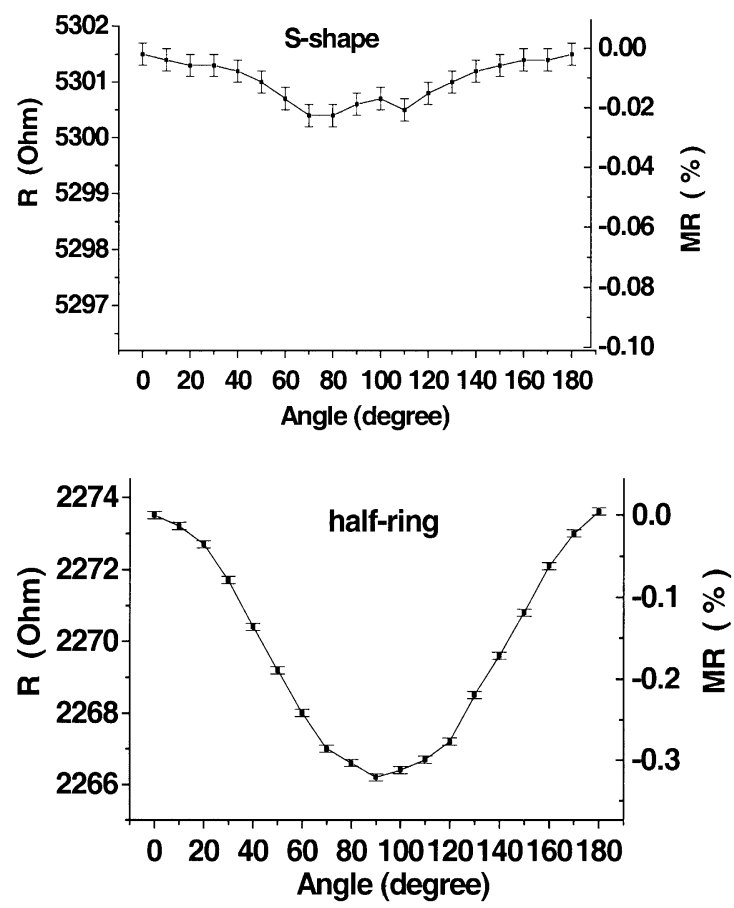

Fig. 2. (a) Magnetoresistance ratio for the $\mathrm{S}$ shape, and (b) magnetoresistance ratio for the half-ring shape in half-ring-inseries $\mathrm{Ni}-\mathrm{Fe}$ wire.
Fig. 2(b), it is roughly $0.33 \%$ which is one order of magnitude larger than that of the S-shape wire. This can be explained by the MFM images at remanence after applying a magnetic field of 6000 Oe (Fig. 3). Black and white poles are clearly observed in the corners formed by the half-ring shape. When the applied magnetic field decreases from $6000 \mathrm{Oe}$, the local magnetization inside the wires starts to rotate towards a direction parallel to the wire due to the shape anisotropy. As the field goes to the negative direction and increases in magnitude, the magnetization becomes unstable towards the negative direction, resulting in a jump in resistance as shown in Fig. 4.

For wires with patterned shape in the octagonin-series, the upper part of Fig. 5 shows the AFM image, whereas the lower part presents the MFM image of the wire. In zero magnetic field, the electrical resistance monotonically decreases roughly from 845 to $530 \Omega$ for temperatures decreasing from 300 to $10 \mathrm{~K}$. It is interesting to compare the magnetoresistance measurements and the MFM images as function of the applied magnetic field. Fig. 6 shows five MFM pictures taken roughly at fields of $0, \pm 180$, and $\pm 900 \mathrm{Oe}$, and the electrical resistance as a function of the applied magnetic field for the octagon-in-series wire. In general, the domain wall magnetoresistance is insensitive to the variation of temperature, whereas the magnetic switching field decreases with increasing temperature. From the magnetoresistance study, we observe that the switch field for the domain wall motion increases with the number of octagons in the wire. It is clear that the behavior of the magnetoresistance of nano-size wires can be related to its different domain configuration.

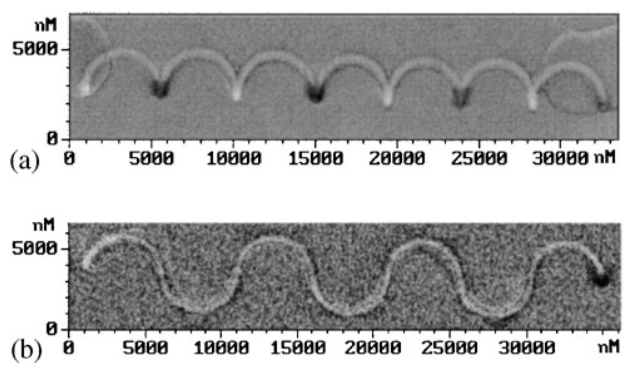

Fig. 3. MFM images for the half-ring-in-series $\mathrm{Ni}-\mathrm{Fe}$ wires. 

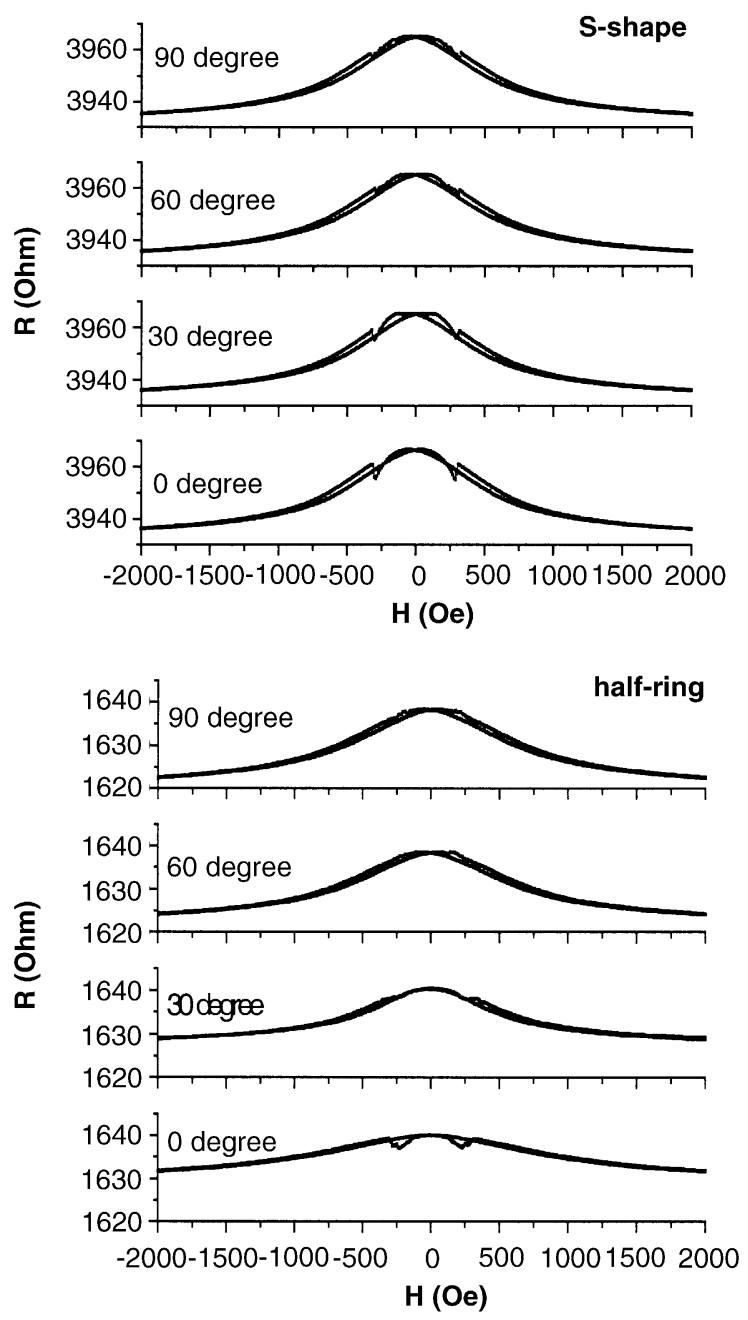

Fig. 4. Magnetoresistance at $77 \mathrm{~K}$ for half-ring-in-series Ni-Fe wires as function of the angle between the magnetic field and wire direction.

The temperature and thickness dependence of the nano-size permalloy zigzag wires with a pitch of $4 \mu \mathrm{m}$ has been reported recently by us $[4,14]$. In these wires, the demagnetizing energy, the shape anisotropy energy, and the exchange energy are always in competition with each other whose competition varies as a function of the applied magnetic field. Here, we would like to discuss in more detail a zigzag wire with a pitch of $8000 \mathrm{~nm}$ and a thickness of $40 \mathrm{~nm}$. Fig. 7 shows the AFM image (upper part), and the MFM image (lower part) of this wire. From the MFM image,

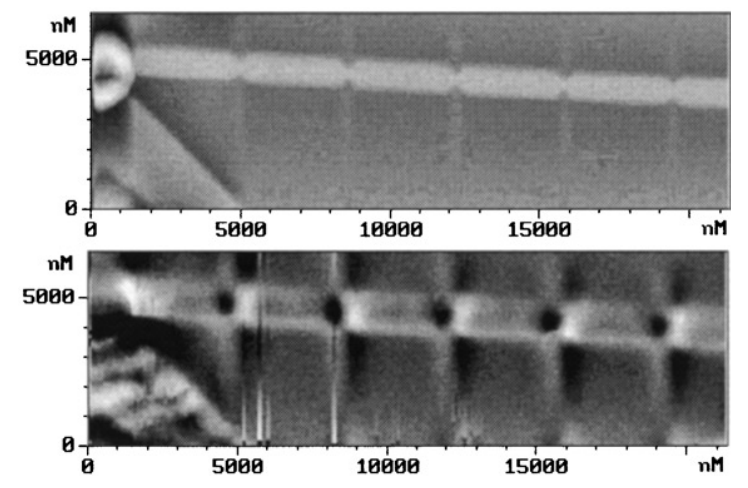

Fig. 5. AFM and MFM images of an octagon-patterned permalloy nano-wire.

alternating black and white areas are found to exist at the corners of the zigzag wire. This means that the shape anisotropy of the segment can create magnetic domain walls. Its number is only a function of the number of zigzags in the wire.

Fig. 8 shows the magnetoresistance ratio as a function of the applied magnetic field in (a) perpendicular and (b) parallel direction. From this figure, both negative and positive domain wall magnetoresistances are found by change of the direction of the applied magnetic field. A negative domain wall magnetoresistance is attributed to the weak localization of the domain wall [15]. A positive domain wall magnetoresistance is the result of the interaction between the spins of the conduction electrons and the local moments inside the domain walls [2]. At analyzing our data for the longitudinal direction of the applied field, we observe a conventional anisotropic magnetoresistance. The decrease in resistance at large fields results from the deviation of the saturated magnetization along the applied field and the current flowing through the circumference of the zigzag wires. However, at a transverse direction of the applied field, vortices form initially at the edge of the zigzag section at small enough field. At further increasing the field along the reverse direction, the vortex disappears and the magnetization becomes parallel to the section. Therefore, the anisotropic magnetoresistance suddenly increases at the reduction of the angle between the magnetization and the current. The parallel state along the circumference will stay until it becomes 


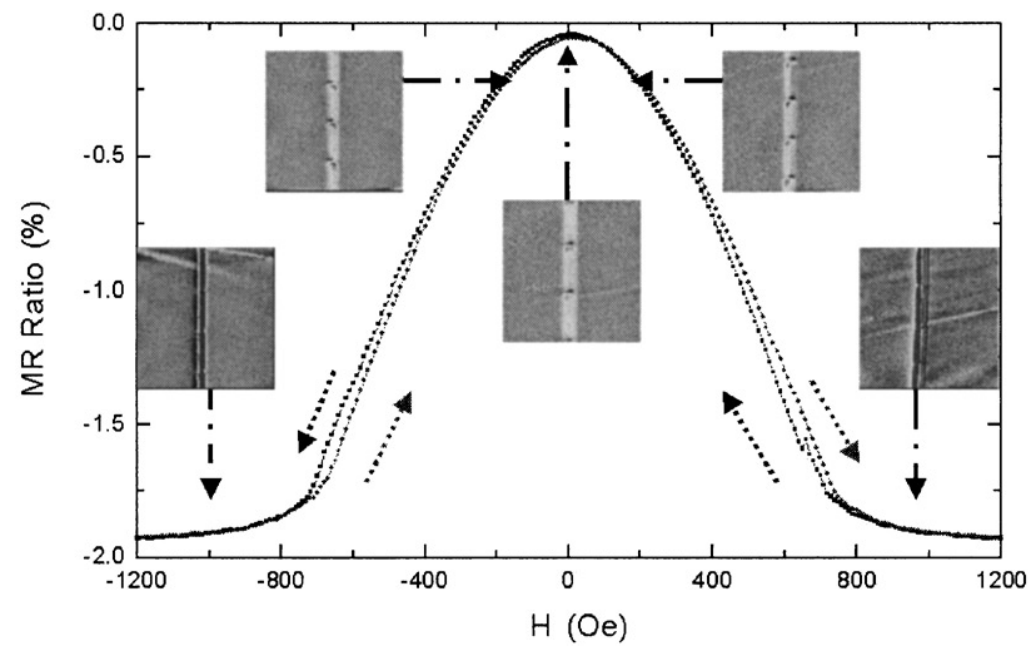

Fig. 6. Five MFM pictures taken roughly at fields of $0, \pm 180$, and $\pm 900 \mathrm{Oe}$, and the magnetoresistance for octagon-in-series Ni-Fe wire.

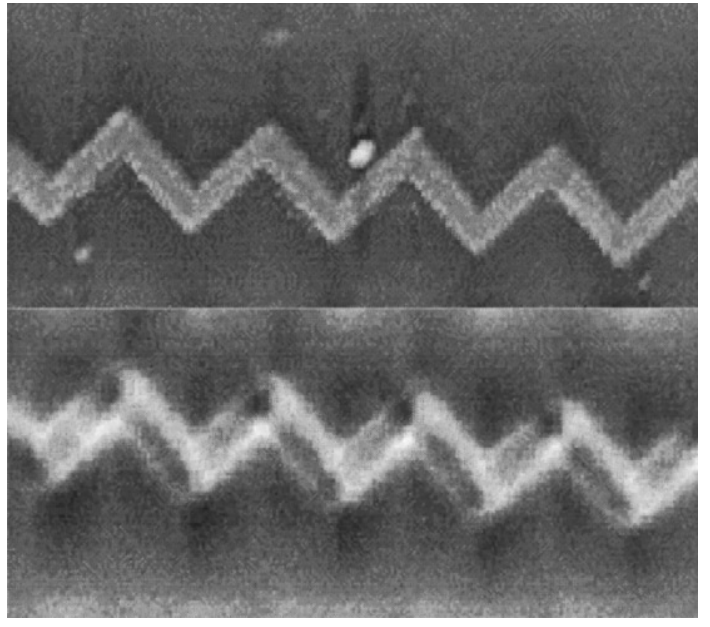

Fig. 7. AFM image (up part), and MFM image (lower part) of the zigzag patterned $\mathrm{Ni}-\mathrm{Fe}$ wire.

unstable at a large enough field and then the resistance will drop again.

In summary, nano-size $\mathrm{NiFe}$ wires with patterned shapes in half-ring-in-series, octagon-inseries, and zigzag-in-series have been successfully fabricated. We have qualitatively reported the relation between the magnetoresistance and the magnetic domain images in these patterned wires. In general, the variation of the magnetoresistance of all patterned nano-size wires studied can be
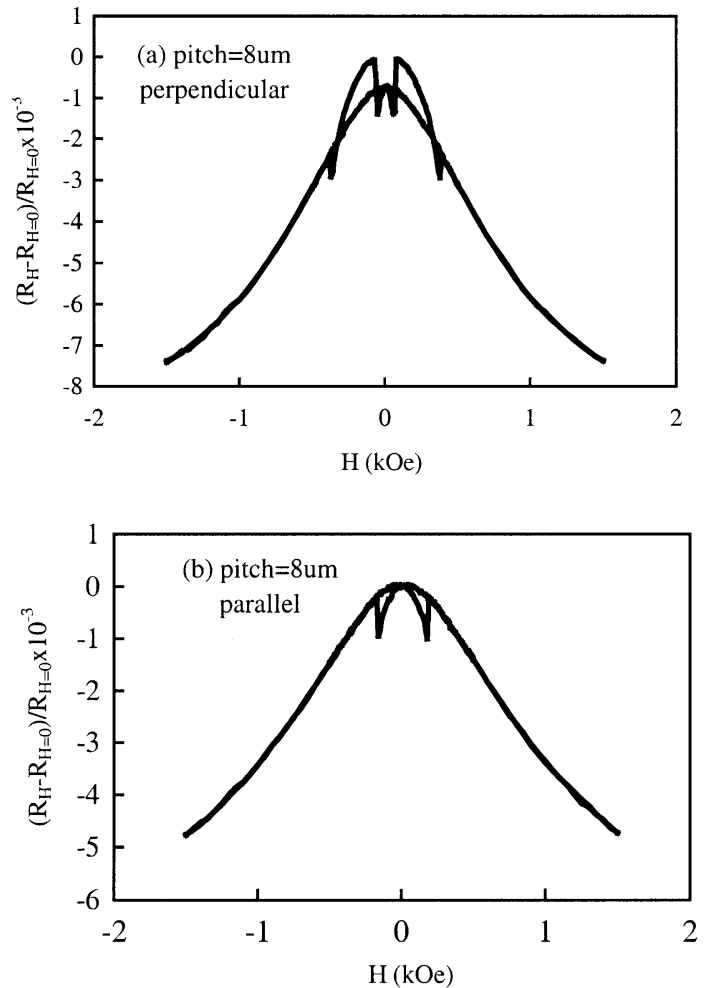

Fig. 8. Magnetoresistance ratio as a function of the applied magnetic field in (a) perpendicular and (b) parallel direction for the zigzag patterned $\mathrm{Ni}-\mathrm{Fe}$ wire.

related to the different domain configurations and explained by the domain switching effect. The number of magnetic domain walls can be 
significantly controlled by the shape anisotropy and size of each section of the patterns to form the wires. Further quantitative analyses will be reported elsewhere.

\section{References}

[1] R.P. Cowburn, D.K. Koltsov, A.O. Adeyeye, M.E. Welland, D.M. Tricker, Phys. Rev. Lett. 83 (1999) 1042.

[2] U. Rudiger, J. Yu, S. Zhang, A.D. Kent, S.S.P. Parkin, Phys. Rev. Lett. 80 (1998) 5639.

[3] S.P. Li, D. Peyrade, M. Natali, A. Lebib, Y. Chen, U. Ebels, L.D. Buda, K. Ounadjela, Phys. Rev. Lett. 86 (2001) 1102.

[4] J.L. Tsai, S.F. Lee, Y.D. Yao, C. Yu, S.H. Liou, J. Appl. Phys. 91 (2002) 7983.

[5] J.G. Zhu, Y. Zheng, G.A. Prinz, J. Appl. Phys. 87 (2000) 6668.
[6] O. Fruchart, J.P. Nozieres, W. Wernsdorfer, D. Givord, F. Rousseaux, D. Decanini, Phys. Rev. Lett. 82 (1999) 1305.

[7] Y. Zheng, J. Zhu, J. Appl. Phys. 81 (1997) 5471.

[8] J. Rothman, M. Kläui, L. Lopez-Diaz, C.A.F. Vaz, A. Bleloch, J.A.C. Bland, Z. Cui, R. Speaks, Phys. Rev. Lett. 86 (2001) 1098.

[9] M. Kläui, J. Rothman, L. Lopez-Diaz, C.A.F. Vaz, J.A.C. Bland, Z. Cui, Appl. Phys. Lett. 78 (2001) 3268.

[10] K. Hong, N. Giordano, Phys. Rev. B 51 (1995) 9855.

[11] U. Ebels, A. Radulescu, Y. Henry, L. Piraux, K. Dunadjela, Phys. Rev. Lett. 84 (2000) 983.

[12] J.F. Gregg, W. Allen, K. Ounadjela, M. Viret, M. Hehn, S.M. Thompson, J.M.D. Coey, Phys. Rev. Lett. 77 (1996) 1580.

[13] T. Taniyama, I. Nakatani, T. Namikawa, Y. Yamazaki, Phys. Rev. Lett. 82 (1999) 2780.

[14] J.L. Tsai, S.F. Lee, Y.D. Yao, C. Yu, J. Magn. Magn. Mater. 239 (2002) 246.

[15] G. Tatara, H. Fukuyama, Phys. Rev. Lett. 78 (1997) 3773. 